\title{
Correlation between the Serum Chloride Levels and the Anion Gap Values in Initial Fluid Resuscitation with Normal Saline in Patients with Diabetic Ketoacidosis at RSUD dr. Saiful Anwar Malang
}

\author{
Hafifa Rahmah Arrazy*, Antonius Freddy**, Nanik Setijowati*** \\ *Resident of Emergency Medicine, Emergency Medicine Science, Universitas Brawijaya, Indonesia \\ ** Teaching Staff of Emergency Medicine, Faculty of Medicine, Universitas Brawijaya, Indonesia \\ *** Teaching Staff of Public Health Science, Faculty of Medicine, Universitas Brawijaya, Indonesia
}

\begin{abstract}
Introduction: Diabetic ketoacidosis (DKA) is characterized by uncontrolled hyperglycemia, ketonemia, and metabolic acidosis. Osmotic diuresis as a result of uncontrolled hyperglycemia causes intracellular dehydration and electrolyte deficits. Excessive production of ketone bodies in the blood instigates high anion gap metabolic acidosis (HAGMA). Fluid resuscitation with normal saline is the initial management of DKA frequently performed at Saiful Anwar Hospital because this infusion fluid is rich in chloride content. Hyperchloremia contributes to the occurrence of normal anion gap metabolic acidosis (NAGMA) after rehydration with large amounts of normal saline because an increase in the chloride levels is not counterbalanced by an increase in the bicarbonate ion concentrations in plasma. Although the anion gap values decrease, acidosis still occurs. A normal anion gap is a recovery sign of DKA. Therefore, research is needed to examine the description and correlation of changes in the serum chloride levels and changes in the anion gap values before and after fluid resuscitation with normal saline. Method: Analytic observational research with cross-sectional approach. A sample of 15 people was collected from July to December 2018 in the emergency department (ED) of Saiful Anwar Hospital.

Results: Significant changes were found in the serum chloride levels $(p=<0.001)$ and in the anion gap values ( $p$ $=0.002$ ) before and after fluid resuscitation with normal saline in patients with DKA. Correlation test results showed a significant relationship between changes in the serum chloride levels and changes in the anion gap values before and after fluid resuscitation with normal saline in patients with DKA $(\mathrm{p}=<0.001, \mathrm{r}=-0.848)$.

Conclusions: Significant changes were found in the serum chloride levels and in the anion gap values before and after fluid resuscitation with normal saline in patients with DKA. The serum chloride levels tended to increase, whereas the anion gap values tended to decrease. Correlation test showed that there was a significant relationship between changes in the serum chloride levels and changes in the anion gap values. The relationship was strong, meaning that the more increasing changes in the serum chloride levels were, the more decreasing changes in the anion gap values would be or vice versa.

Keywords: The chloride level, the anion gap, normal saline, $0.9 \% \mathrm{NaCl}$, diabetic ketoacidosis, changes in chloride, changes in the anion gap, correlation between chloride and the anion gap.

DOI: $10.7176 / \mathrm{JHMN} / 66-10$

Publication date:September $30^{\text {th }} 2019$

\section{Introduction}

Diabetic ketoacidosis (DKA) is one of the most serious and acute complications of diabetes. DKA is a significant contributor to mortality, costs, and poor quality of life. Approximately 100,000 patients with diabetic ketoacidosis are admitted to hospitals per year in the United States. The total incidence of DKA tends to increase, mainly due to an increase in type 2 diabetes cases and obesity. ${ }^{1,2,3}$

DKA consists of the biochemical triad of hyperglycemia, ketonemia, and metabolic acidosis resulting from absolute or relative insulin deficiency and an increase in counter-regulatory hormones. These life-threatening consequences produce severe body water deficit, the accumulation of ketone bodies, and electrolyte disturbances. ${ }^{2,4}$

Hyperglycemia develops because of three processes: increased gluconeogenesis, accelerated glycogenolysis, and impaired glucose utilization by peripheral tissues. The hyperglycemia will lead to glycosuria and osmotic diuresis, resulting in hyperosmolar intracellular dehydration. In DKA, fluid deficits may be up to $10 \%$ of total body weight and electrolyte deficits in the body, one of which is chloride.

In DKA, lipolysis resulting in high circulating levels of free fatty acids may occur. These free fatty acids are taken up by the liver and oxidized to ketone bodies. Excessive production of ketone bodies in the anionic form can cause high plasma anion gap. Ketone bodies are relatively strong acids that dissociate at physiologic $\mathrm{pH}$. The dissociation in the form of $\mathrm{H}+$ ions will be buffered by bicarbonate. When the plasma bicarbonate concentrations decrease, a high anion gap acidosis metabolic (HAGMA) develops. ${ }^{1,2,4,5,6}$
\end{abstract}


The anion gap calculation formula is $\left[\mathrm{Na}^{+}-\left(\mathrm{Cl}^{-}+\mathrm{HCO}_{3}^{-}\right)\right]$. Based on diagnostic criteria, the anion gap value in DKA $>10$. The increased anion gap is a predictor of metabolic acidosis, and the degree of metabolic acidosis can be estimated by the size of the anion gap. ${ }^{1,7}$

Initial management of adult patients with DKA according to the clinical pathway at Saiful Anwar Hospital is rehydration with normal saline fluid $(0.9 \% \mathrm{NaCl})$ intravenously and insulin administration (when the serum potassium levels $<3.3 \mathrm{mEq} / \mathrm{L}$ ). The initial dose of normal saline infusion is $15-20 \mathrm{~mL} / \mathrm{kg} /$ hour over the first hour. Subsequent amount of fluid given depends on the hydration status, hemodynamic conditions, and electrolyte levels. Electrolyte levels are monitored after 2 hours of initial fluid resuscitation.

The goals of rapid rehydration are to improve the circulatory volume and tissue perfusion, clear ketone bodies, and correct electrolyte imbalances. Normal saline contains $154 \mathrm{mEq} / \mathrm{L}$ chloride and $154 \mathrm{mEq} / \mathrm{L}$ sodium. The chloride concentration of saline is higher than that of human plasma $(97-107 \mathrm{mEq} / \mathrm{L}) .8,9,10$

Normal saline $(0.9 \%$ sodium chloride) is a low-cost and easy-to-find crystalloid fluid which becomes fluid choice by doctors in some cases. However, normal saline is now under observation due to concerns about hyperchloremic metabolic acidosis, oliguria, prolonged acidosis, and coagulopathy. Consistent data are found regarding increased serum chloride levels associated with complications of acidosis, AKI, or electrolyte disturbances after being given at least 2 liters of normal saline. An increase in the chloride levels is not counterbalanced by an increase in the bicarbonate ion concentrations in plasma. Although the anion gap values decrease, acidosis still occurs. Thus, normal anion gap metabolic acidosis (NAGMA) or hyperchloremic acidosis arises. $9,10,11$

Blood glucose level $<200 \mathrm{mg} / \mathrm{dL}$, serum bicarbonate level $>15 \mathrm{mEq} / \mathrm{L}, \mathrm{pH}>7.3$, and normal anion gap are recovery signs of DKA. ${ }^{12}$

This research was conducted to examine the description and correlation of changes in the serum chloride levels and changes in the anion gap values before and after initial fluid resuscitation with normal saline in patients with DKA in the ED of Saiful Anwar Hospital and to examine the correlation between changes in the serum chloride levels and changes in the anion gap values.

Aim

To examine the description and correlation of changes in the serum chloride levels and changes in the anion gap values before and after fluid resuscitation with normal saline in patients with DKA.

\section{Method}

This research employed analytic observational research with cross-sectional approach to examine correlation between changes in the serum chloride levels and changes in the anion gap values before and after fluid resuscitation with normal saline in patients with DKA. The research population was all hyperglycemic crisis patients in the emergency department of Saiful Anwar Hospital. The research samples were 15 people in accordance with the inclusion criteria (all patients with DKA with the serum glucose level $\geq 250 \mathrm{mg} / \mathrm{dL}$, arterial $\mathrm{pH}<7.3$, the anion gap $>10$, the serum bicarbonate level $<18 \mathrm{mEq} / \mathrm{L}$, and ketonemia) from medical records during the research period. Chloride, bicarbonate, and sodium levels were recorded and the anion gap values were calculated before and after fluid resuscitation with normal saline.

\section{Results}

The research began from January to March 2018 with a sample of 15 patients with DKA who received initial fluid resuscitation with normal saline in the ED of Saiful Anwar Hospital. Data were collected prospectively from medical records from July to December 2018 in accordance with research ethics.

a. Characteristics of the Research

Characteristics of the sample in this research are presented in Table 1 below.

Table 1. Characteristics of the research subjects

\begin{tabular}{llll}
\hline \multicolumn{2}{l}{ Characteristic } & $\mathbf{n}$ & $\mathbf{( \% )}$ \\
\hline Age & $\leq 50$ year & 9 & 60,3 \\
& $\geq 50$ year & 6 & 39,7 \\
\hline \multirow{2}{*}{ Total } & & 15 & 100 \\
\hline Sex & Male & 6 & 40 \\
& Female & 9 & 60 \\
\hline Total & & 15 & 100 \\
\hline
\end{tabular}


b. Description of changes in the serum chloride levels and the anion gap values before and after fluid resuscitation with normal saline

Before a test on this numerical data was conducted, its normality was determined first. The Shapiro-Wilk test was employed because of the sample size $<40$. From the test results, all data were normally distributed $(p>0.05)$, except for data of the anion gap values before fluid resuscitation with normal saline which was not normally distributed $(\mathrm{p}<0.05)$. Data transformation was then conducted for non-normally distributed data. It turned out that the anion gap values before fluid resuscitation with NS remained not normally distributed ( $p<0.05$ ). Next, all normally distributed data were analyzed using parametric test, while non-normally distributed data were analyzed using non-parametric test.

Study data showed changes in the serum chloride levels and the anion gap values. The average value of the serum chloride levels increased by $11.3 \mathrm{mmol} / \mathrm{L}$ and the average value of the anion gap values decreased by $7.89 \mathrm{mmol} / \mathrm{L}$. Data analysis results with Paired T-Test showed significant changes in the serum chloride levels before and after fluid resuscitation with normal saline in patients with DKA $(p=<0.001)$. Data analysis results with the Wilcoxon test also showed significant changes in the anion gap values before and after fluid resuscitation with normal saline in patients with DKA $(\mathrm{p}=0.002)$.

c. Correlation between changes in the serum chloride levels and changes in the anion gap values before and after fluid resuscitation with normal saline

Based on the Shapiro-Wilk test for normality, changes in the serum chloride levels and changes in the anion gap values showed a normal distribution $(\mathrm{p}>0.05)$, the linearity requirements were fulfilled as illustrated in Graph 1 . The relationship could be explained by around $71.88 \%$, while the remaining $28.12 \%$ was due to other factors. Because all variables were normally distributed, data could be analyzed using the Pearson's correlation test. Test results showed that there was a significant relationship between changes in the serum chloride levels and changes in the anion gap values before and after resuscitation with normal saline fluid in patients with DKA $(\mathrm{p}<0.001, \mathrm{r}=$ $-0.848)$. The relationship was very strong $(>0.8)$, meaning that the more increasing changes in the serum chloride levels were, the more decreasing changes in the anion gap values would be or vice versa.

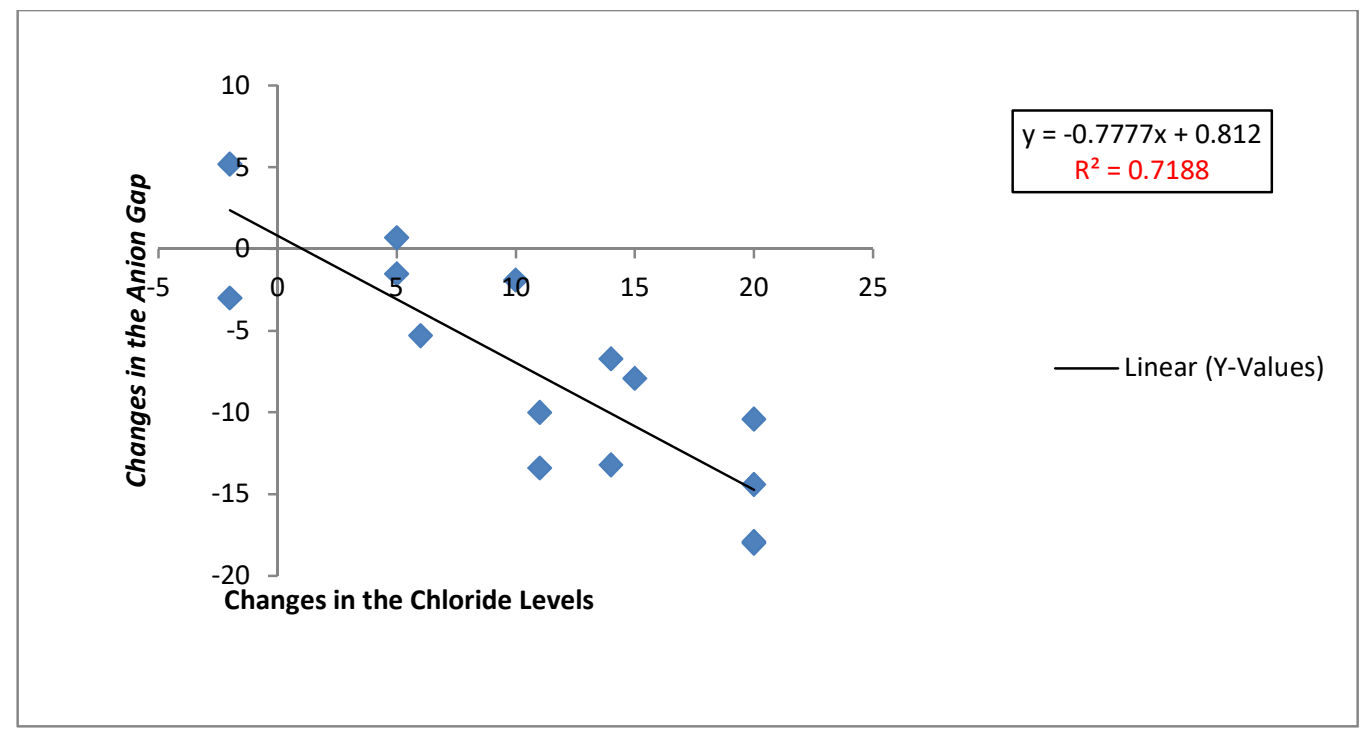

Graph 1. Linearity between changes in the serum chloride levels and changes in the anion gap values before and after fluid resuscitation with normal saline.

\section{Discussion}

In this research, it was found that changes in the serum chloride levels tended to increase after fluid resuscitation with normal saline. It happened because the research subjects received fluids with high chloride content.

It is in line with Barker et al. (2015) who conducted a review of 4 studies regarding the comparison of normal saline infusion, lactated ringer, and plasmalyte in the acute care setting with different cases. All of these studies showed hyperchloremia after fluid resuscitation with normal saline infusion. Consistent data revealed that some complications in the group receiving 2 liters of normal saline infusion were associated with increased serum chloride levels. Hyperchloremia can cause acidosis, AKI, and increased resource utilization if not corrected immediately. 
On the other hand, changes in the anion gap values tended to decrease after fluid resuscitation with normal saline.

HAGMA is a characteristic of DKA. Initial management of DKA includes rapid rehydration with normal saline as an effort to cleanse the ketones bodies from the body through urinary excretion and an effort to inhibit lipolysis by decreasing counterregulatory hormones through intravenous insulin administration. With the decreasing number of ketone bodies in the blood, the anion gap value will decrease as well.

Correlation test results on changes in the two variables above showed a strong relationship.

When examined through the calculation formula of the anion gap value $\{\mathrm{AG}=\mathrm{Na}+-(\mathrm{Cl}-+\mathrm{HCO} 3-)\}$, with the sodium value considered unchanged and the $\mathrm{Cl}$ - and $\mathrm{HCO} 3-$ levels are low in DKA before receiving fluid resuscitation, the results of the anion gap calculation become high. After receiving fluid resuscitation with normal saline, the $\mathrm{Cl}$ level might increase, $\mathrm{HCO} 3$ - level might slightly increase, and the anion gap value might decrease. Changes in the sodium, chloride, and bicarbonate values will be able to influence the anion gap calculation results.

Before DKA treatment, low chloride levels are common and seem to be a compensatory response to HAGMA in DKA. After fluid resuscitation with normal saline in the first hours, the excess of unmeasured anions is quickly replaced by measured anions (chloride). A decrease in the anion gap value reflects a reduced number of unmeasured anions (especially ketone bodies) which seems to show recovery signs of DKA. Barker et al. found a research analysis on the amount of normal saline infusion fluid which directly correlates with hyperchloremic metabolic acidosis levels. It happens because the anion gap values become normal due to an increase in the chloride levels even though acidosis still occurs as the result of bicarbonate dilution after normal saline infusion administration into the body.

\section{Conclusions}

1. Significant changes were found in the serum chloride levels and in the anion gap values before and after fluid resuscitation with normal saline in patients with DKA. The serum chloride levels tended to increase, whereas the anion gap values tended to decrease.

2. There was a significant relationship between changes in the serum chloride levels and changes in the anion gap values before and after resuscitation with normal saline fluid in patients with DKA. The relationship was very strong $(>0.8)$, meaning that the more increasing changes in the serum chloride levels were, the more decreasing changes in the anion gap values would be or vice versa.

\section{Suggestions}

1. The effects of hyperchloremia and metabolic acidosis may have been examined in this research sample. It is expected that further studies will examine the clinical impact on patients with DKA in Saiful Anwar Hospital.

\section{References}

1. Kitabchi AE, Umpierrez GE, Miles JM, Fisher JN. 2009. Hyperglycemia Crises in Adult Patients with Diabetes. Diabetes Care; 32(7): 1335-43.

2. Mahler SA, Conrad SA, Wang H, Arnold TC, 2011. Resuscitation with balanced electrolyte solution prevents hyperchloremic metabolic acidosis in patients with diabetic ketoacidosis. American Journal of Emergency Medicine; 29: 670-674.

3. Desai D, Mehta D, Mathias P, Menon G, Schubart UK. 2018. Health Care Utilization and Burden of Diabetic Ketoacidosis in the U.S. Over the Past Decade: A Nation Wide Analysis. Care. diabetesjournals.org

4. Nyenwe, E. A. Kitabchi, A. E. 2016. The evolution of diabetic ketoacidosis: An update of its etiology, pathogenesis and management, Metabolism: Clinical and Experimental. Elsevier Inc., 65(4): 507-521.

5. Tran TT. 2017. Review of evidence for Adult Diabetic Ketoacidosis Management Protocols. Frontiers in endocrinology. 8(106);1-13.

6. Eledrisi MS, Alshanti MS, Shah Faiq, Blorosy B, Jaha N. 2006. Overview of the Diagnosis and Management of Diabetic Ketoacidosis. Am J Med Sci; 331(5):243-251.

7. Guyton AC, Hall JE. 2012. Acid-Base Regulation. Guyton and Hall Textbook of Medical Physiology. ECG Medical Publisher; 13(31): 406. 
8. Nigam A. 2010. Hyperglycemic Emergencies: Evaluation and Management Guidelines. Medicine Update; 2(24): 147-154.

9. Self WH. 2018. Balanced Crystalloids versus Saline in Noncritically Ill Adults. N Engl J Med; 378:819-28.

10. Barker ME. 2015. 0,9\% saline induced hyperchloremic acidosis. Journal of Trauma Nursing; 22(2): 112116.

11. Bandak G, Kashani KB. 2017. Chloride in intensive care units: a key electrolyte [version 1; referees: 3 approved]. Review. F1000 research.

12. Tintinalli. 2016. Tintinalli's Emergency Medicine: A Comprehensive Study Guide, USA, 8th ed., Mc Graw Hill Education. 